\title{
The Tobacco Metabolite NNK Enhances Pancreatic Cancer Cell Stemness and Chemoresistance by Activating the B2AR-Akt Autophagy Axis
}

\section{Xin Chen}

The first affiliated hospital of xi'an jiaotong university

\section{Weifan Zhang}

The First Affiliated Hospital of Xi'an Jiaotong University

Rujuan Liu

The First Affiliated Hospital of Xi'an Jiaotong University

\section{Zeen Zhu}

The First Affiliated Hospital of Xi'an Jiaotong University

\section{Mengyuan Gong}

The First Affiliated Hospital of Xi'an Jiaotong University

\section{Qiqi Wang}

The First Affiliated Hospital of Xi'an Jiaotong University

\section{Weikun Qian}

The First Affiliated Hospital of Xi'an Jiaotong University

\section{Zheng Wu}

The First Affiliated Hospital of Xi'an Jiaotong University

\section{Qingyong Ma}

The First Affiliated Hospital of Xi'an Jiaotong University

Zheng Wang ( $\sim$ zheng.wang11@mail.xjtu.edu.cn )

Xi'an Jiaotong University Medical College First Affiliated Hospital Department of Hepatobiliary Surgery https://orcid.org/0000-0002-0490-466X

\section{Research}

Keywords: NNK, Pancreatic cancer, Autophagy, Stemness, Chemoresistance

Posted Date: October 14th, 2021

DOI: https://doi.org/10.21203/rs.3.rs-882597/v2

License: (a) (i) This work is licensed under a Creative Commons Attribution 4.0 International License. Read Full License 


\section{Abstract}

Background: Low responsiveness to chemotherapy is an important cause of poor prognosis in pancreatic cancer. Smoking is a high-risk factor for pancreatic cancer and its resistance to gemcitabine; however, the underlying mechanisms remain unclear. 4-(Methylnitrosamino)-1-(3-pyridyl)-1-butanone (NNK) is the main metabolite of tobacco burning and has been shown to be associated with cancer development and chemoresistance, but in pancreatic cancer, its mechanism remains poorly understood.

Methods: The effect of NNK on pancreatic cancer cell viability was confirmed by using Cell Counting Kit-8 and colony formation assays. Stem cell sphere formation assays and western blotting/qPCR measurements of stemness-related molecules were used to detect pancreatic cancer cell stemness. The pancreatic cancer autophagy status was evaluated by immunofluorescence staining of LC3 and western blotting/qPCR analysis of autophagy-related molecules.

Results: NNK promoted stemness and gemcitabine resistance in pancreatic cancer cell lines. Furthermore, NNK intervention increased autophagy and changed the expression levels of autophagy-related markers, which preliminarily confirmed the activation of autophagy by NNK. Finally, the results showed that NNKpromoted stemness, and gemcitabine resistance was activated by the autophagy pathway, which was mediated by the $\beta 2 A R-A k t$ signalling pathway.

Conclusions: Autophagy induced by activating the NNK-induced $\beta 2 A R-A k t$ signalling pathway promoted stemness and gemcitabine resistance in pancreatic cancer cells.

\section{Background}

Pancreatic cancer is one of the most malignant tumours due to its late diagnosis, aggressive tumour growth and high level of metastasis, and the overall 5-year survival rate of patients with pancreatic cancer is less than $10 \%{ }^{[1]}$. Pancreatic ductal adenocarcinoma (PDAC) is the most common pathological type of pancreatic cancer, accounting for more than $90 \%$ of cases ${ }^{[2,3]}$. Due to insidious onset, some patients have lost the chance of surgical resection when they are diagnosed, and chemotherapy is the main treatment. Gemcitabine is the most common first-line chemotherapy drug approved for the treatment of advanced PDAC, either alone or in combination with other chemotherapeutic agents ${ }^{[4]}$. Although gemcitabine combined with other chemotherapy agents can profoundly improve the prognosis of advanced pancreatic cancer, the development of chemoresistance still leads to poor clinical outcomes $^{[5]}$. Therefore, it is particularly important to further elucidate the mechanisms of gemcitabine resistance and to find a therapeutic method to reverse it.

Smoking is the first investigated modifiable risk factor for pancreatic cancer development. Epidemiological results show that smokers have a significantly increased risk of developing pancreatic cancer and a worse prognosis than nonsmokers ${ }^{[6]}$. 4-(Methylnitrosamino)-1-(3-pyridyl)-1-butanone (NNK), a nitrosamine, which is the main metabolite produced by burning tobacco, is thought to have a strong 
carcinogenic effect ${ }^{[7]}$. Interestingly, NNK promotes the proliferation ${ }^{[8,9]}$, migration ${ }^{[10]}$, and stemness ${ }^{[11]}$ of pancreatic cancer cells, and there is a significant correlation between cell stemness and gemcitabine resistance. However, the functional roles and mechanisms of NNK in the sensitivity of pancreatic cancer to gemcitabine treatment remain poorly understood and thus need to be further clarified.

Autophagy is phagocytosis of cytoplasmic proteins or organelles, which are encapsulated into vesicles and fused with lysosomes to form autophagy lysosomes followed by degradation of encapsulated contents to achieve the metabolic needs of cells and the renewal of some organelles ${ }^{[12]}$. Although the mechanisms of autophagy are not fully understood, studies have shown that it is closely related to chemotherapy resistance ${ }^{[13,14]}$. The chloroquine (CQ) autophagy blocker also significantly improves the sensitivity of pancreatic cancer cells to gemcitabine.

Beta-adrenergic receptor ( $\beta A R)$, a member of the G-protein-coupled receptor family, is paid more attention recently. Three subtypes, $\beta 1, \beta 2$ and $\beta 3$, have been identified. $\beta A R$ is thought to be involved in the biological behaviours of pancreatic cancer, especially $\beta 2 A R$, which mediates chronic stress and its development ${ }^{[15]}$. In addition, $\beta$ AR also activates protein kinase $A(P K B) / A k t$ through phosphatidylinositol 3-kinase (PI3K), thereby activating a series of transcription factors to promote the proliferation of pancreatic cancer cells ${ }^{[16]}$.

In this study, we attempted to investigate the contributions and mechanisms of NNK in the resistance of pancreatic cancer cells to gemcitabine. We demonstrated that NNK promotes pancreatic cancer cell resistance to gemcitabine and stemness through increased autophagic activity, which is mediated by the B2AR-Akt signalling pathway.

\section{Materials And Methods}

\section{Cell culture and reagents}

The BxPC-3 and Panc-1 human pancreatic cancer cell lines were purchased from the Chinese Academy of Sciences Cell Bank of Type Culture Collection (CBTCCCAS, Shanghai, China). The cells were cultured in RPMI-1640 or DMEM (Gibco, Thermo Fisher Scientific, USA) supplemented with 10\% foetal bovine serum (FBS) at $37^{\circ} \mathrm{C}$ with $5 \% \mathrm{CO}_{2}$. NNK (a proper concentration for cells), CQ (10 $\mu \mathrm{M}$ for cells), and gemcitabine (Gem, $30 \mu \mathrm{M}$ for cells) were purchased from Sigma-Aldrich (St. Louis, MO, USA), ICI 118551 (30 $\mu \mathrm{M}$ for cells), LY294002 (30 $\mu \mathrm{M}$ for cells) and U0126 (30 $\mu \mathrm{M}$ for cells) were purchased from MedChem Express (Monmouth Junction, NJ, USA); the inhibitors were dissolved in DMSO according to the manufacturer's protocol.

\section{Cell Counting Kit-8}

Cell viability was measured using Cell Counting Kit-8 (CCK-8) according to the manufacturer's protocol. Briefly, 1000 pancreatic cancer cells were seeded in 96-well plates and incubated for the indicated times. Before testing, $10 \mu \mathrm{l}$ of CCK-8 solution was added to each well of the plate. The plate was incubated for 4 
h at $37^{\circ} \mathrm{C}$, and the optical density (OD) was measured at $450 \mathrm{~nm}$ using a multifunctional microplate reader (POLARstar OPTIMA; BMG, Offenburg, Germany).

\section{Colony formation assay and stem cell sphere formation assay}

Pancreatic cancer cells were seeded into 6-well plates at 2000 cells per well for $24 \mathrm{~h}$ and treated with different concentrations of drugs for 2 weeks. Then, the 6-well plates were washed with phosphatebuffered saline (PBS), fixed with $4 \%$ paraformaldehyde, and stained with crystal violet solution for 20 min. The number of colonies was counted in five random fields by microscopy under 100x magnification. In addition, after harvesting, resuspension and counting, 1000 cells were seeded into ultralow attachment 6-well plates at 1000 cells per well for serum-free DMEM/F12 medium (containing $20 \mathrm{ng} / \mathrm{ml}$ EGF, 20 $\mathrm{ng} / \mathrm{ml} \mathrm{FGF}$ and $1 \%$ B27) and cultured for 1 week. The number of stem cell spheres was counted under a microscope (Nikon Instruments Inc.).

\section{siRNA, RNA extraction and qRT-PCR}

Pancreatic cancer cells were seeded into 6-well plates $24 \mathrm{~h}$ before transfection. siRNA (Gene Pharma) and Lipofectamine 8000 Transfection Reagent (Invitrogen, Carlsbad, CA, USA) were used according to the protocols. Total RNA was extracted from cultured pancreatic cancer cells using TRIzol reagent purchased from Invitrogen (Carlsbad, CA, USA). cDNA was reverse transcribed using a cDNA synthesis kit (TaKaRa, Tokyo, Japan). Quantitative PCR was performed using SYBR qPCR Master Mix (TaKaRa, Tokyo, Japan). The mRNA expression level for each sample was determined using the $2^{-\Delta \Delta c t}$ method with three biological replicates for comparative qRT-PCR.

\section{Immunofluorescence assay}

Pancreatic cancer cells were cultivated on circular glass slides in 24-well dishes after treatment, fixed in $4 \%$ paraformaldehyde for 20 min, washed with PBS twice, treated with permeabilization solution ( $1 \%$ Triton X-100 in PBS), washed with PBS again, and blocked with $5 \%$ bovine serum albumin (BSA, SigmaAldrich, Germany) for $1 \mathrm{~h}$. Samples were incubated with primary antibodies in PBS buffer overnight at $4^{\circ} \mathrm{C}$. Then, the samples were washed with PBS twice and incubated with fluorescein isothiocyanateconjugated AffiniPure goat anti-rabbit IgG secondary antibody (dilution, 1:300; Beijing Zhongshan Golden Bridge Biotechnology, China) for $60 \mathrm{~min}$ at room temperature. After the samples were stained with DAPI, images were captured with a ZEISS Instruments confocal microscope.

\section{Western blot analysis}

Pancreatic cancer cells were seeded in 6-well plates for $24 \mathrm{~h}$ and then treated with various interventions. Then, protein lysates were obtained by incubating the cells in RIPA buffer containing proteinase inhibitors for $10 \mathrm{~min}$ on ice and centrifugation at $12000 \mathrm{~g}$ for $15 \mathrm{~min}$ at $4^{\circ} \mathrm{C}$. The samples were separated by $10 \%$ SDS-PAGE and transferred to PVDF membranes. After blocking with $5 \%$ nonfat milk, the membranes were incubated with primary antibodies (LC3, ATG5, ATG7, Beclin1 SOX2, OCT4, Nanog, $\beta 1$ AR, B2AR, Akt, p-Akt; 
$\beta$-actin; all purchased from Cell Signalling Technology, USA) at $4^{\circ} \mathrm{C}$ overnight. Next, the membranes were washed with PBST buffer 4 times and incubated with peroxidase-conjugated secondary antibodies for 1 $\mathrm{h}$ at room temperature. After washing with PBST buffer 4 times, the membranes were visualised with an ECL chemiluminescent detection system (Bio-Rad, USA).

\section{Autophagy Detection Using mRFP-GFP Adenoviral Vector}

Ad-mRFP-GFP-LC3 was purchased from HanBio, Shanghai, China. and the experiment was carried out according to the provided instructions. Cells were plated in 6-well dishes and were grown to $50 \%$ confluency at the time of infection. Then, the cells were cultured in DMEM supplemented with $1 \%$ FBS and adenoviruses for $2 \mathrm{~h}$ at $37^{\circ} \mathrm{C}$. After infection, cells were grown for $48 \mathrm{~h}$ in medium with $10 \% \mathrm{FBS}$ and used for further study. Autophagy was observed under a fluorescence microscope. Autophagic flux was determined by evaluating the number of GFP- and RFP-positive puncta.

\section{Statistical analysis}

All statistical analyses were performed by GraphPad Prism 6.0 software (GraphPad, La Jolla, CA, USA). All data were presented as the mean \pm SD (standard deviation) of triplicate samples. Differences with a $P$ value $<0.05$ were statistically significant. Differences between two groups were analysed by independent sample t-tests, and differences among multiple groups were analysed by one-way ANOVA; *represents $\mathrm{P}$ $<0.05$, **represents $\mathrm{P}<0.01$ and ***represents $\mathrm{P}<0.001$.

\section{Results}

\section{NNK could strengthen stemness and chemoresistance in pancreatic cancer cells.}

To address the potential role of NNK in pancreatic cancer cells, we first treated BxPC-3 and Panc-1 cells with NNK and found that NNK significantly increased the colony formation ability of pancreatic cancer cells (Fig. 1A), which meane that NNK may have a pro-stemness effect on pancreatic cancer cells. To further confirm this hypothesis, we then examined the effect of NNK on pancreatic cancer spherogenesis, and the results showed that the number of pancreatic cancer stem cell spheres (PCSCs) formed in the NNK-treated group was significantly higher than that in the control group (Fig. 1B). Since SRY-Box Transcription Factor 2 (SOX-2), Octamer-Binding Protein 4 (OCT-4) and Nanog Homeobox (Nanog) are three common indicators of cell stemness in pancreatic cancer cells, we examined their mRNA and protein expression levels in control group and NNK-treated PCSCs group. The results showed that SOX-2, OCT-4 and Nanog were all significantly upregulated by NNK stimulation (Fig. 1C). In summary, the above findings suggest that NNK could strengthen the stemness of pancreatic cancer cells.

Increasing evidence suggested that cell stemness is often accompanied by an increase in chemoresistance in pancreatic cancer. Hence, we next aimed to verify whether NNK is associated with gemcitabine resistance in pancreatic cancer cells. We performed a CCK-8 assay and found that pancreatic cancer proliferation was significantly decreased by gemcitabine, while this phenomenon was 
partly eliminated by NNK (Fig. 1D). Additionally, using a colony formation assay, we confirmed the above findings (Fig. 1E). In summary, the above findings suggest that NNK could promote the chemoresistance of pancreatic cancer cells.

\section{NNK could induce autophagy in pancreatic cancer cells.}

Notably, the activation of autophagy and related pathways may play an essential role in cancer cell stemness and chemotherapy resistance. Hence, we aimed to determine whether NNK influences autophagy in pancreatic cancer. Firstly, we used immunofluorescence to stain LC3 autophagy spots and found that NNK significantly increased the number of LC3 autophagy spots in Panc-1 cells (Fig. 2A). In addition, western blotting also showed that NNK can influence the protein expression levels of LC3 and p62 which were belong to representative cell autophagy markers in a time- and dose-dependent manner (Fig. 2B). It seemed that NNK may be preliminarily related to autophagy. Secondly, we applied the mRFPGFP-LC3 autophagy double-labelled adenovirus system to observe the effect of NNK on autophagy more intuitively and accurately. After $24 \mathrm{~h}$, fluorescence microscopy revealed that the red and green autophagy spots in the transfected cells were significantly increased after NNK intervention compared with that in the control group, and the yellow spots formed by the overlap of red and green spots were also significantly increased. In addition, the proportion of single red spots in cells in the NNK intervention group was significantly higher than that in the control group (Fig. 2C), which suggested that NNK promotes autophagy again. Thirdly, we examined the effect of NNK on autophagy-related pathways and found that NNK obviously modulated the mRNA expression of autophagy-related 5 (ATG5), autophagyrelated 7 (ATG7), and Beclin1 in a time- and dose-dependent manner (Fig. 2D). Moreover, these phenomena were also confirmed by western blotting (Fig. 2E). Finally, to further analyse the role of the above autophagy-related pathways in NNK-induced autophagy, siRNA was used to knock down the expression ATG5, ATG7, and Beclin (Fig. 2F). Western blotting results showed that the increase in LC3 levels caused by NNK was inhibited by si-ATG5, ATG7 and Beclin1. Additionally, the number of LC3 autophagy spots in cells was also reduced (Fig. 2G). In conclusion, the above findings indicated that NNK could promote autophagy in pancreatic cancer cells in an ATG5-, ATG7- and Beclin1-dependent manner.

\section{NNK could enhance the stemness and chemoresistance of pancreatic cancer cells by activating autophagy.}

Notably, researchers have demonstrated that the stemness and chemoresistance of cancer cells were related to cell autophagy. Hence, to determine whether the increase in stemness/chemoresistance of pancreatic cancer cells induced by NNK was due to its effect on cell autophagy, we conducted a series of experiments.

We firstly conducted colony formation assays and transwell assays to evaluate the influence of autophagy on pancreatic cancer single-cell malignancy/stem cell-like behaviour with NNK treatment. The results showed that $\mathrm{CQ}$, a classic autophagy inhibitor, decreased the number of Panc-1 colonies, indicating that autophagy may benefit the colony formation ability of single pancreatic cancer cells (Fig. 3A). In addition, NNK-induced promotion of Panc-1 colonies can also be significantly inhibited by CQ. 
Moreover, Transwell assay also confirmed similar phenomena in the invasion/migration of single pancreatic cancer cells (Fig. 3B); hence, the above findings suggest that NNK-mediated strengthening of stem cell-like behaviour in pancreatic cancer cells is autophagy dependent. Next, to illustrate the effect of NNK and downstream autophagy in pancreatic cancer cells stemness more directly, we measured the spherogenesis of Panc-1 cells, and the results showed that CQ inhibited sphere formation. NNK-induced promotion of Panc-1 spherogenesis was also significantly inhibited by CQ (Fig. 3C). We also found the similar variation as above in the expression of SOX-2, OCT-4 and Nanog stemness markers (Fig. 3D). In summary, the previous results revealed that NNK may enhance the stemness of pancreatic cancer cells by activating autophagy.

We next examined whether NNK-induced pancreatic cancer chemoresistance was mediated by cell autophagy. By using the CCK-8 assay, we found that autophagy inhibition sensitized the killing effect of gemcitabine on pancreatic cancer cells, and this phenomenon was evident even in the presence of NNK (Fig. 3E). Moreover, the colony formation assay also suggested the same phenomenon (Fig. 3F). These findings revealed that NNK enhanced the chemoresistance of pancreatic cancer cells by activating autophagy

\section{NNK could promote autophagy through the modulation of $\beta 2 A R$ and Akt.}

We previously suggested that NNK is an analogue of catecholamines that can activate $\beta A R$ efficiently, so we hypothesized that NNK promoted autophagy by activating the $\beta A R$ signaling pathway in pancreatic cancer cells. To test this hypothesis, a series of experiments was designed. Firstly, $\beta 2 A R$ (the main isoform of $\beta A R$ expressed in pancreatic cancer) was efficiently knocked down by using a specific siRNA (Fig. 4A). Immunofluorescence analysis revealed that $\beta 2 A R$ knockdown significantly reduced LC3 autophagy spots (induced by NNK) in pancreatic cancer cells (Fig. 4B), and a similar effect was also confirmed by examining the expression of ATG5, ATG7 and Beclin1 autophagy markers (Fig. 4C), which suggests that the autophagy-promoting effect of NNK partly depends on $\beta 2 A R$.

As a $G$ protein-coupled receptor embedded the cell membrane, $\beta A R$ senses the stimulation of extracellular ligands such as NNK and transduction signals through various intracellular pathways. Next, we aimed to determine the possible downstream autophagy-related pathways of $\beta A R$ after stimulation with NNK. Through continuous screening and testing, we found that Akt, an important pro-pancreatic cancer kinase, may mediate this process. To verify this hypothesis, we used immunofluorescence and western blotting and found that knockdown of Akt significantly reduced LC3 autophagy spots and LC3 protein levels (induced by NNK) in PC cells (Fig. 4D, E). Moreover, a similar effect was also confirmed by examining the expression of the ATG5, ATG7 and Beclin1 autophagy markers (Fig. 4F). These inhibitory functions were similar to those of LY294002 Akt inhibitor (a PI3K inhibitor that can block Akt activation), which suggested that the autophagy-promoting effect of NNK was partly dependent on Akt.

\section{NNK could form a potential $\beta 2 A R-A k t$ feedback loop in pancreatic cancer cells autophagy.}


The above findings suggested that the autophagy-promoting effect of NNK was $\beta 2 A R$ - and Aktdependent. Next, clearly elucidating the up- and downstream relationship between $\beta 2 A R-A k t$ and NNKinduced pancreatic cancer cells autophagy was critical to our research. In general, membrane receptors are the initiators of signalling pathways when the cell is externally stimulated. Hence, we hypothesised that Akt may be downstream of $\beta 2 A R$. To test this, we first aimed to exclude the influence of $\beta 1 A R$ on NNK-induced pancreatic cancer $\beta A R$ activation, and the results showed that $\beta 1$ AR knockdown (Fig. 5A) had no effect on the activation of Akt (Fig. 5B); however, $\beta 2 A R$ knockdown and inhibition significantly suppressed Akt activation (phosphorylation) and LC3 maturation (Fig. 5B, C), which suggested that the B2AR-Akt axis was the main pancreatic cancer cells autophagy inducer after NNK stimulation. However, we accidentally discovered that $\beta 2 A R$ expression was modulated by NNK in pancreatic cancer cells (Fig. $5 \mathrm{C})$, which reminded us that there may be a potential $\beta 2 A R$-Akt-autophagy feedback loop underlying the effects of NNK. To explore this hypothesis, we treated Panc-1 and BxPC-3 cells with NNK for different time periods and found that the mRNA expression of $\beta 2 A R$ (rather than $\beta 1 A R$ ) was increased in a timedependent manner (Fig. 5D), and this trend can be blocked by inhibitors (Fig. 5E). Moreover, at the protein level, NNK also promoted $\beta 2$ AR expression and Akt activation (Fig. 5F). In conclusion, these findings suggested that NNK activates autophagy through the $\beta 2 A R-A k t$ pathway and increases $\beta 2 A R$ expression levels to further strengthen this autophagy activation process, thus forming a potential $\beta 2 A R-A k t$ feedback loop in pancreatic cancer cells autophagy.

\section{Discussion}

Pancreatic cancer is one of the most lethal malignancies, with a five-year survival rate of less than $10 \%{ }^{[1]}$. The incidence of pancreatic cancer is increasing each year, but due to late diagnosis and the rapid progression of the disease, only a small number of patients can receive radical excision after a clear diagnosis ${ }^{[17]}$. Chemotherapy is also a treatment for patients with advanced pancreatic cancer, and drugs such as gemcitabine are first-line effective treatments in management. Gemcitabine was approved for clinical use in 1995 by inhibiting DNA synthesis and blocking the progression of the G1/S stage of the cell cycle ${ }^{[18]}$. Clinically, gemcitabine resistance often occurs, leading to failure of first-line chemotherapy. Therefore, it is important to explore the mechanism of gemcitabine resistance. 4-(Methylnitrosamino)-1(3-pyridyl)-1-butanone (NNK) is one of the byproducts of burning tobacco and has been reported to be related to drug resistance against various chemotherapeutic drugs ${ }^{[19-21]}$. Cancer stem cells(CSCs) are closely related to the generation of drug resistance, their self-renewal function, formation/maintenance of the CSCs phenotype and cytoprotective machinery, allowing CSCs to survive after drug exposure ${ }^{[22]}$. Moreover, autophagy has been shown to play an important role in many biological properties of pancreatic cancer cells, including survival, dormancy and epithelial-mesenchymal transition(EMT), metastasis, and gemcitabine resistance ${ }^{[23]}$. However, the relationship between NNK, autophagy, stemness, and gemcitabine resistance in pancreatic cancer remains unclear.

Therefore, in the present study, we aimed to determine the effect of NNK on stemness and gemcitabine resistance. First, we used the Panc-1 and BxPC-3 pancreatic cancer lines for our experiments and 
demonstrated that NNK promotes cell growth and sphere formation. Moreover, NNK increases the expression levels of OCT-4, SOX-2, and Nanog, which are thought to play an important role in tumour stem cells. It is consistent with previous research that elevated levels may indicate increased stemness in tumour cells ${ }^{[23-25]}$. Our experiments also confirmed that NNK can promote gemcitabine resistance. Interestingly, NNK may promote both stemness and gemcitabine resistance, which was ultimately seen as a challenge for pancreatic cancer treatment. Since LC3 and p62 detection by western blotting or immunofluorescence has become an acceptable method for monitoring autophagy and autophagyrelated processes ${ }^{[26,27]}$, our results showed that NNK can change the expression of LC3 and p62, which indicated that NNK can indeed promote the production of autophagy. We further confirmed that NNK promoted the expression of ATG5, ATG7, and Beclin1, and after knockdown of these autophagy-related genes, LC3 expression also changed accordingly. Thus, we hypothesized that NNK promotes stemness and gemcitabine resistance in pancreatic cancer cells by activating autophagy. We confirmed this through conducting experiments with $\mathrm{CQ}$, an autophagy inhibitor that mainly inhibits autophagy by impairing autophagosome fusion with lysosomes ${ }^{[28]}$.

Finally, we tried to determine the molecular mechanism by which NNK promotes autophagy. $\beta-A R$ is a member of the G-protein-coupled receptor family, and intracellular signalling after $\beta$-AR activation is largely modulated through cyclic adenosine monophosphate (CAMP) and protein kinase A (PKA), triggering the activation of a series of transcription factors and promoting the expression of related genes $^{[29]}$. Several studies have shown that $\beta-A R$ is closely related to autophagy, but whether it promotes or inhibits autophagy is not completely consistent across studies ${ }^{[30-32]}$. NNK is a $\beta$-AR agonist ${ }^{[33]}$, and our experiments demonstrate that NNK activates autophagy through $\beta 2$-AR but not $\beta 1$-AR. Protein kinase $\mathrm{B}(\mathrm{PKB}) /$ Akt protein kinase is a serine/threonine protein kinase with high homology to both PKA and PKC. On the one hand, it can be activated by upstream $\beta 2-\mathrm{AR}^{[34-36]}$; on the other hand, the activation of Akt is also associated with autophagy ${ }^{[37]}$. Then, we demonstrated that NNK promotes autophagy by activating $\beta 2-A R$ to phosphorylate Akt. Moreover, we also found that this process can increase the expression of $\beta 2-$ AR, further enhancing autophagy. However, we have not proven how Akt activates autophagy.

\section{Conclusions}

In summary, we discovered that NNK promotes human pancreatic cancer stemness and gemcitabine resistance. We also demonstrated the mechanism by which NNK regulates the $\beta 2 A R-A k t$ signalling pathway, which activates autophagy. This may explain the mechanism by which smoking promotes gemcitabine resistance, but further mechanisms should be explored.

\section{Abbreviations}

PDAC: Pancreatic ductal adenocarcinoma

NNK: 4-(Methylnitrosamino)-1-(3-pyridyl)-1-butanone 
CQ: chloroquine

$\beta A R:$ Beta-adrenergic receptor

PKB/Akt: protein kinase A

CCK-8: Cell Counting Kit-8

PCSCs: cancer stem cell spheres

SOX-2: SRY-Box Transcription Factor 2

OCT-4: Octamer-Binding Protein 4

Nanog: Nanog Homeobox

ATG5: Autophagy-related 5

ATG7: Autophagy-related 7

EMT: Epithelial-mesenchymal transition

\section{Declarations}

\section{Ethics approval and consent to participate}

Studies involving animals was approved by the Ethical Committee of the First Affiliated Hospital of Xi'an Jiaotong University, Xi'an, China.

\section{Consent for publication}

We have obtained consent to publish from the participants to report individual data.

\section{Availability of data and materials}

All data generated or analyzed during this study are available from the corresponding author on reasonable request.

\section{Conflict of Interest Statement}

The authors declare that there were no commercial or financial relationships that could be construed as a potential conflict of interest.

\section{Funding}

This study was supported by National Natural Science Foundation of China (NSFC 81872008, 82072702); National Key Research and Development Program of China (2019YFC1315900, Sub program, 
2019YFC1315902); Clinical Research Award of the First Affiliated Hospital of Xi'an Jiaotong University, China (No. XJTU1AF-CRF-2019-005); The Science and Technology Innovation as a Whole Plan Project of Shaanxi Province, China. (No. 2016KJZDSF01-05-01).

\section{Authors' Contributions}

X.C. and W.Z performed most of the experiments, treated data, and wrote the manuscript; R.L., M.G., Z.Z. and Q.W. participated in analyzing the data and organized the Figures; W.Q. and Z.W read and reviewed the manuscript. Z.W. and Q.M. designed the experiment and manuscript. All authors read and approved the final manuscript.

\section{Acknowledgements}

Not applicable.

\section{References}

1. Siegel RL, Miller KD, Fuchs HE, Jemal A. Cancer Statistics, 2021[J]. CA Cancer J Clin. 2021;71(1):733.

2. Siersema PD. Pancreatic Cancer Awareness Issue 2019[J]. Endoscopy. 2019;51(11):1009.

3. Klein AP. Pancreatic cancer: a growing burden[J]. Lancet Gastroenterol Hepatol. 2019;4(12):895-6.

4. Sarvepalli D, Rashid MU, Rahman AU, Ullah W, Hussain I, Hasan B, et al. Gemcitabine: A Review of Chemoresistance in Pancreatic Cancer[J]. Crit Rev Oncog. 2019;24(2):199-212.

5. Zeng S, Pottler M, Lan B, Grutzmann R, Pilarsky C, Yang H. Chemoresistance in Pancreatic Cancer[J]. Int J Mol Sci. 2019;20(18): 4504.

6. Zanini S, Renzi S, Limongi AR, Bellavite P, Giovinazzo F, Bermano G. A review of lifestyle and environment risk factors for pancreatic cancer[J]. Eur J Cancer. 2021;145:53-70.

7. Hoffmann D, Hecht SS. Nicotine-derived N-nitrosamines and tobacco-related cancer: current status and future directions[J]. Cancer Res. 1985;45(3):935-44.

8. Zhang D, Lei J, Ma J, Chen X, Sheng L, Jiang Z, et al. beta2-adrenogenic signaling regulates NNKinduced pancreatic cancer progression via upregulation of HIF-1alpha[J]. Oncotarget. 2016;7(14):17760-72.

9. Zhang D, Ma Q, Wang Z, Zhang M, Guo K, Wang F, et al. beta2-adrenoceptor blockage induces G1/S phase arrest and apoptosis in pancreatic cancer cells via Ras/Akt/NFkappaB pathway[J]. Mol Cancer. 2011;10:146.

10. Pham H, Chen M, Takahashi H, King J, Reber HA, Hines OJ, et al. Apigenin inhibits NNK-induced focal adhesion kinase activation in pancreatic cancer cells[J]. Pancreas. 2012;41(8):1306-15.

11. Nimmakayala RK, Seshacharyulu P, Lakshmanan I, Rachagani S, Chugh S, Karmakar S, et al. Cigarette Smoke Induces Stem Cell Features of Pancreatic Cancer Cells via PAF1[J]. Gastroenterology. 2018;155(3):892-908 e6. 
12. Glick D, Barth S, Macleod KF. Autophagy: cellular and molecular mechanisms[J]. J Pathol. 2010;221(1):3-12.

13. Li YJ, Lei YH, Yao N, Wang CR, Hu N, Ye WC, et al. Autophagy and multidrug resistance in cancer[J]. Chin J Cancer. 2017;36(1):52.

14. Piffoux $\mathrm{M}$, Eriau E, Cassier PA. Autophagy as a therapeutic target in pancreatic cancer[J]. $\mathrm{Br} \mathrm{J}$ Cancer. 2021;124(2):333-44.

15. Hefner J, Csef H, Kunzmann V. [Stress and pancreatic carcinoma--beta-adrenergic signaling and tumor biology][J]. Dtsch Med Wochenschr. 2014;139(7):334-8.

16. Schaal C, Chellappan SP. Nicotine-mediated cell proliferation and tumor progression in smokingrelated cancers[J]. Mol Cancer Res. 2014;12(1):14-23.

17. Ansari D, Tingstedt B, Andersson B, Holmquist F, Sturesson C, Williamsson C, et al. Pancreatic cancer: yesterday, today and tomorrow[J]. Future Oncol. 2016;12(16):1929-46.

18. Mini E, Nobili S, Caciagli B, Landini I, Mazzei T. Cellular pharmacology of gemcitabine[J]. Ann Oncol. 2006;17 Suppl 5:v7-12.

19. Lee TY, Liu CL, Chang YC, Nieh S, Lin YS, Jao SW, et al. Increased chemoresistance via Snail-Raf kinase inhibitor protein signaling in colorectal cancer in response to a nicotine derivative[J]. Oncotarget. 2016;7(17):23512-20.

20. Leslie EM, Ghibellini G, Nezasa K, Brouwer KL. Biotransformation and transport of the tobaccospecific carcinogen 4-(methylnitrosamino)-1-(3-pyridyl)-1-butanone (NNK) in bile duct-cannulated wild-type and Mrp2/Abcc2-deficient (TR ) Wistar rats[J]. Carcinogenesis. 2007;28(12):2650-6.

21. Gordon W, Galitovskiy V, Edwards R, Andersen B, Grando SA. The tobacco carcinogen nitrosamine induces a differential gene expression response in tumour susceptible $\mathrm{A} / \mathrm{J}$ and resistant $\mathrm{C} 3 \mathrm{H}$ mouse lungs[J]. Eur J Cancer. 2013;49(3):725-33.

22. Kabakov A, Yakimova A, Matchuk O. Molecular Chaperones in Cancer Stem Cells: Determinants of Stemness and Potential Targets for Antitumor Therapy[J]. Cells. 2020;9(4).

23. Usman RM, Razzaq F, Akbar A, Farooqui AA, Iftikhar A, Latif A, et al. Role and mechanism of autophagy-regulating factors in tumorigenesis and drug resistance[J]. Asia Pac J Clin Oncol. 2020.

24. Zhang Q, Han Z, Zhu Y, Chen J, Li W. The Role and Specific Mechanism of OCT4 in Cancer Stem Cells: A Review[J]. Int J Stem Cells. 2020;13(3):312-25.

25. Shariati F, Favaedi R, Ramazanali F, Ghoraeian P, Afsharian P, Aflatoonian B, et al. Increased expression of stemness genes REX-1, OCT-4, NANOG, and SOX-2 in women with ovarian endometriosis versus normal endometrium: A case-control study[J]. Int J Reprod Biomed. 2018;16(12).

26. Tanida I, Ueno T, Kominami E. LC3 and Autophagy[J]. Methods Mol Biol. 2008;445:77-88.

27. Zaffagnini G, Savova A, Danieli A, Romanov J, Tremel S, Ebner M, et al. p62 filaments capture and present ubiquitinated cargos for autophagy[J]. The EMBO journal. 2018;37(5). 
28. Mauthe M, Orhon I, Rocchi C, Zhou X, Luhr M, Hijlkema KJ, et al. Chloroquine inhibits autophagic flux by decreasing autophagosome-lysosome fusion[J]. Autophagy. 2018;14(8):1435-55.

29. Johnson M. Molecular mechanisms of beta(2)-adrenergic receptor function, response, and regulation[J]. J Allergy Clin Immunol. 2006;117(1):18-24; quiz 5.

30. Aranguiz-Urroz P, Canales J, Copaja M, Troncoso R, Vicencio JM, Carrillo C, et al. Beta(2)-adrenergic receptor regulates cardiac fibroblast autophagy and collagen degradation[J]. Biochim Biophys Acta. 2011;1812(1):23-31.

31. Cammalleri M, Locri F, Catalani E, Filippi L, Cervia D, Dal Monte M, et al. The Beta Adrenergic Receptor Blocker Propranolol Counteracts Retinal Dysfunction in a Mouse Model of Oxygen Induced Retinopathy: Restoring the Balance between Apoptosis and Autophagy[J]. Front Cell Neurosci. 2017;11:395.

32. Deng J, Jiang P, Yang T, Huang M, Xie J, Luo C, et al. beta2adrenergic receptor signaling promotes neuroblastoma cell proliferation by activating autophagy[J]. Oncol Rep. 2019; ;42(4):1295-1306.

33. Askari MD, Tsao MS, Schuller HM. The tobacco-specific carcinogen, 4-(methylnitrosamino)-1-(3pyridyl)-1-butanone stimulates proliferation of immortalized human pancreatic duct epithelia through beta-adrenergic transactivation of EGF receptors[J]. J Cancer Res Clin Oncol. 2005;131(10):639-48.

34. Zhang C, Liao X, Ma Z, Liu S, Fang F, Mai H. Overexpression of beta-Adrenergic Receptors and the Suppressive Effect of beta2-Adrenergic Receptor Blockade in Oral Squamous Cell Carcinoma[J]. J Oral Maxillofac Surg. 2020;78(10):1871 e1- e23.

35. Yun JH, Jeong HS, Kim KJ, Han MH, Lee EH, Lee K, et al. beta-Adrenergic receptor agonists attenuate pericyte loss in diabetic retinas through Akt activation[J]. FASEB J. 2018;32(5):2324-38.

36. Chu SY, Peng F, Wang J, Liu L, Meng L, Zhao J, et al. Catestatin in defense of oxidative-stressinduced apoptosis: A novel mechanism by activating the beta2 adrenergic receptor and PKB/Akt pathway in ischemic-reperfused myocardium[J]. Peptides. 2020;123:170200.

37. Park CH, Lee IS, Grippo P, Pandol SJ, Gukovskaya AS, Edderkaoui M. Akt kinase mediates the prosurvival effect of smoking compounds in pancreatic ductal cells[J]. Pancreas. 2013;42(4):655-62.

\section{Figures}


A
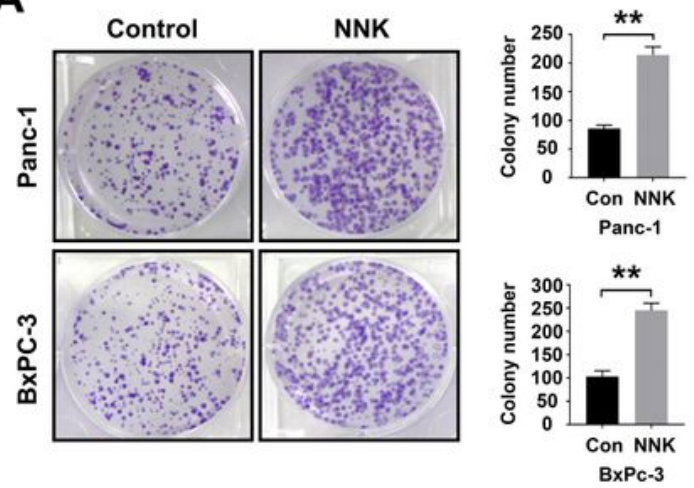

C

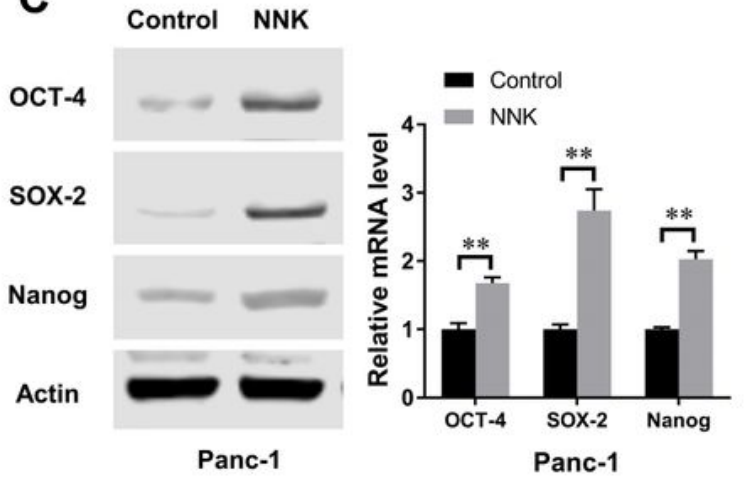

B
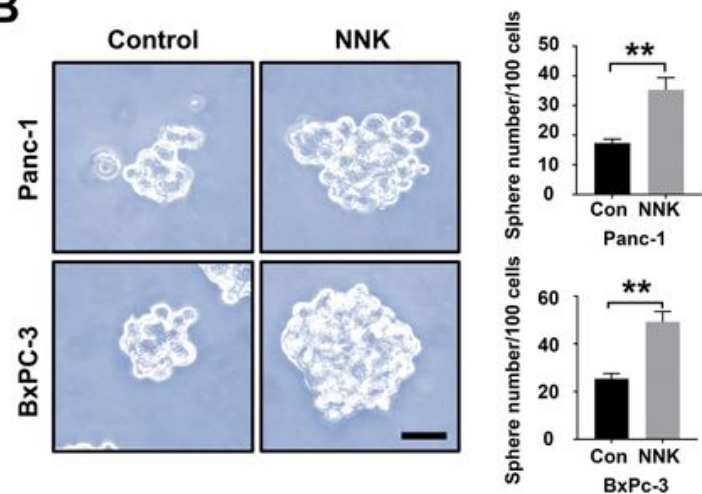

D

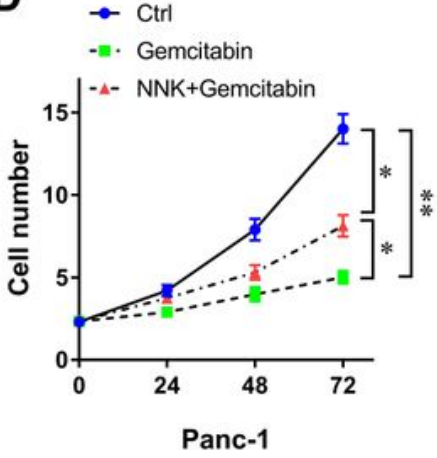

$\rightarrow$ Ctrl

-... Gemcitabin

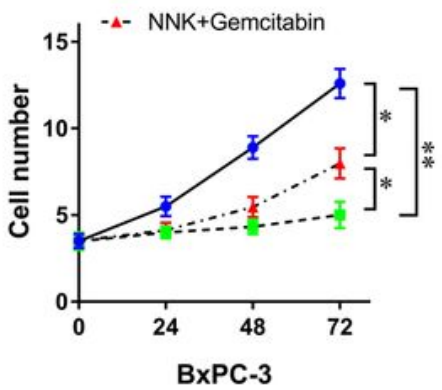

E
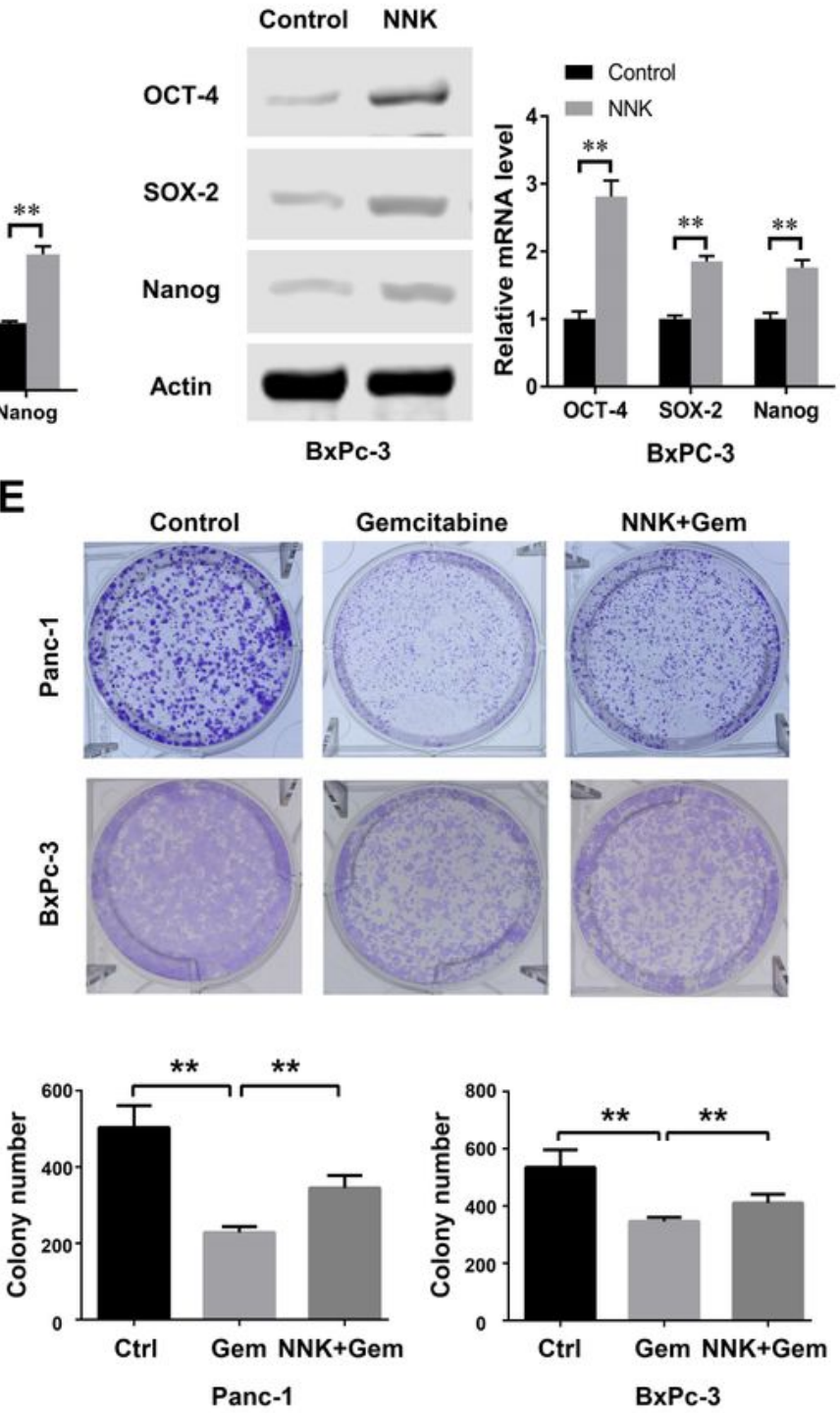

\section{Figure 1}

NNK strengthens stemness and chemoresistance in pancreatic cancer cells. Results of the Panc-1/BxPC3 cell colony formation assay (A) and stem cell sphere formation assay (B) in the control group and NNKtreated group are shown. (C) The mRNA and protein expression levels of OCT-4, SOX-2 and Nanog were determined in the control group and NNK-treated group of Panc-1/BxPC-3 cells. CCK-8 assays (D) and 
colony formation assays were conducted $(E)$ in the control group, gemcitabine group and gemcitabine plus NNK group. (*p $\left.<0.05,{ }^{* *} p<0.01\right)$

A
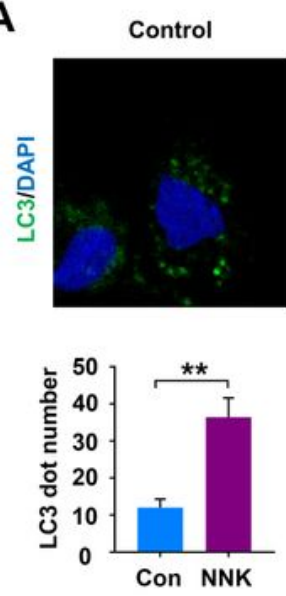

C

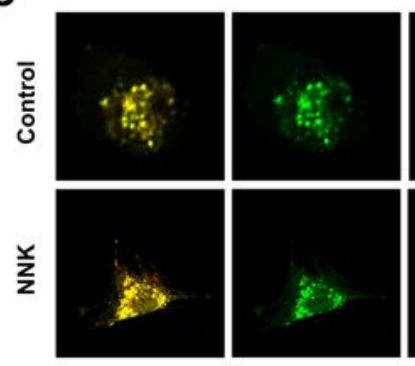

D

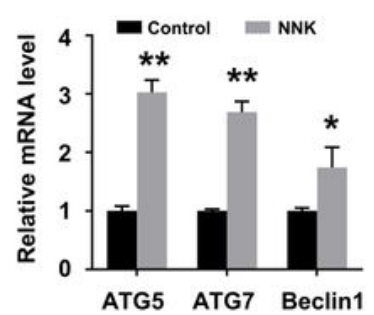

F

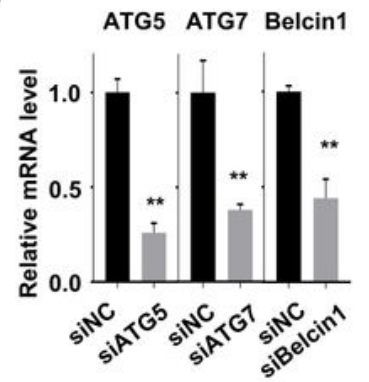

B
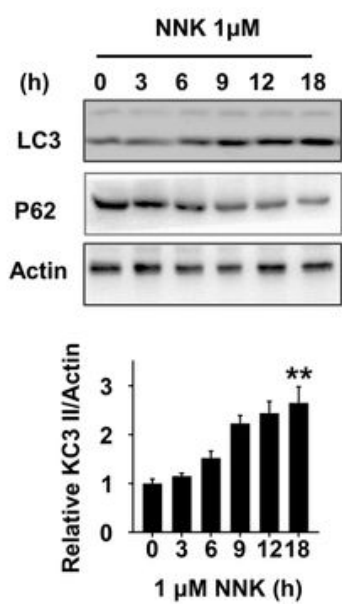

$-\mathrm{mRFP}=\mathrm{GFP}$

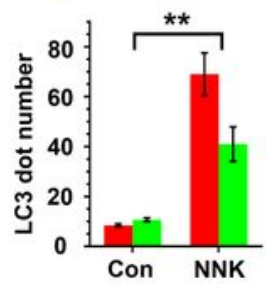

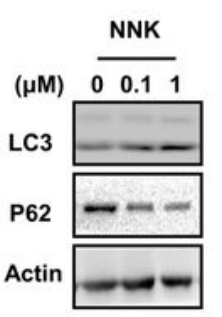

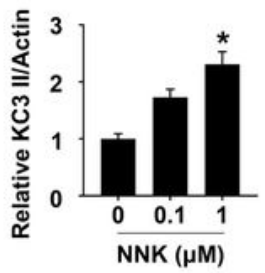

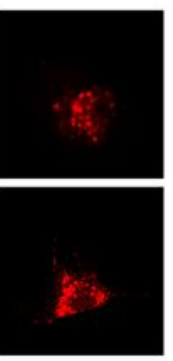

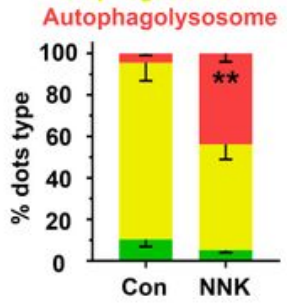

E

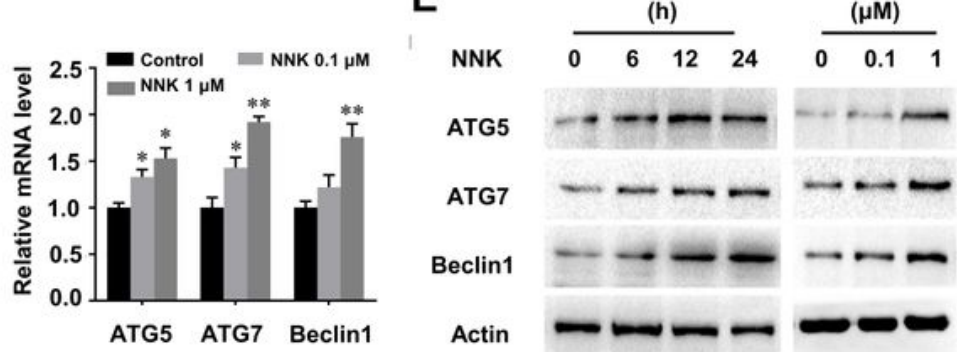

G
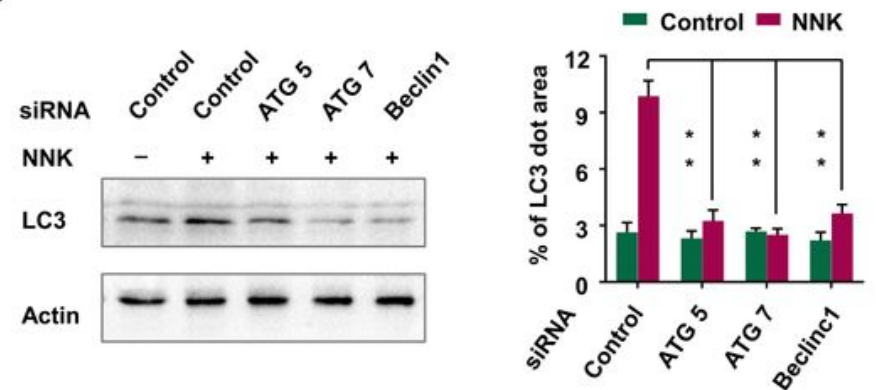

Figure 2

NNK induces autophagy in pancreatic cancer cells. (A) Immunofluorescence shows LC3 autophagy spots in the control group and NNK group of Panc-1 cells and a statistical graph. (B) The protein levels of LC3 and p62 were determined in Panc-1 cells at different doses and time periods of NNK treatment. (C) The 
MRFP-GFP-LC3 autophagy double-labelled adenovirus system shows autophagosomes (red spots) and autophagolysosomes (yellow spots) in the control group and NNK group of Panc- 1 cells and a statistical graph of the results is shown. (D)(E) The mRNA and protein levels of ATG5, ATG7 and Beclin1 in the control group and NNK group of Panc-1 cells were determined. (F) The mRNA levels of ATG5, ATG7 and Beclin1 were reduced after treatment with siRNA. (G) western blotting and immunofluorescence analyses show that the protein level and LC3 autophagy spots caused by NNK are blocked by ATG5, ATG7, and Beclin1 knockdown. (*p $\left.<0.05,{ }^{*} \mathrm{p}<0.01\right)$.

A
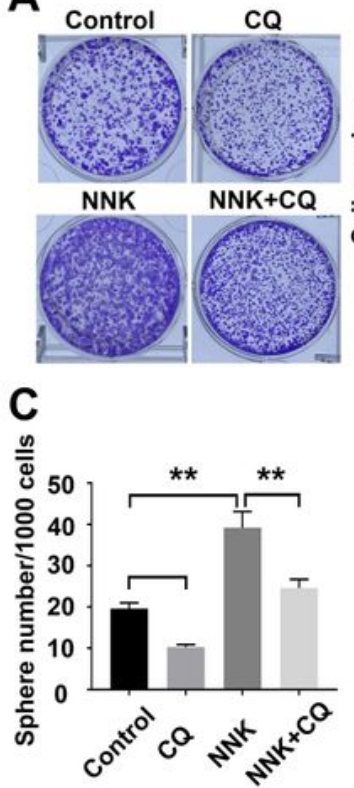
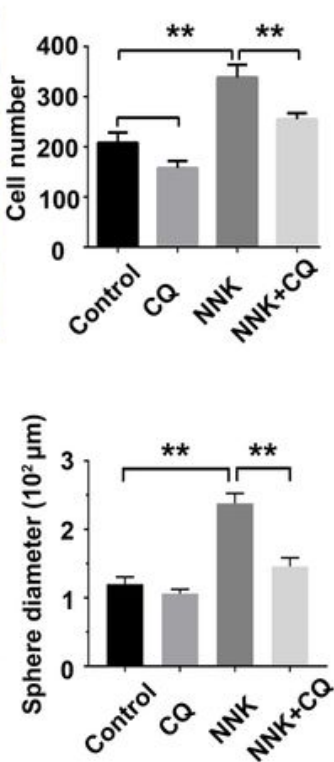

D

- Control
$\mathrm{CQ}$

- NNK

NNK $+\mathrm{CQ}$

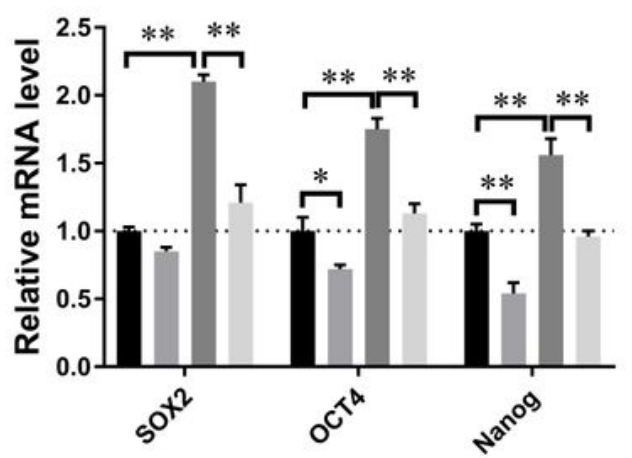

$\mathbf{F}$
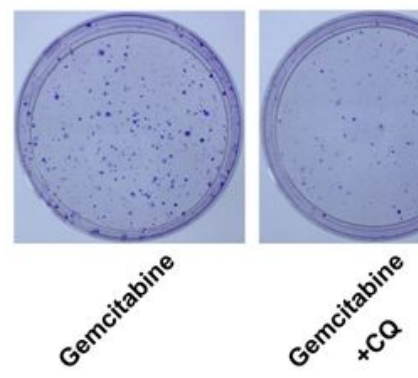

B
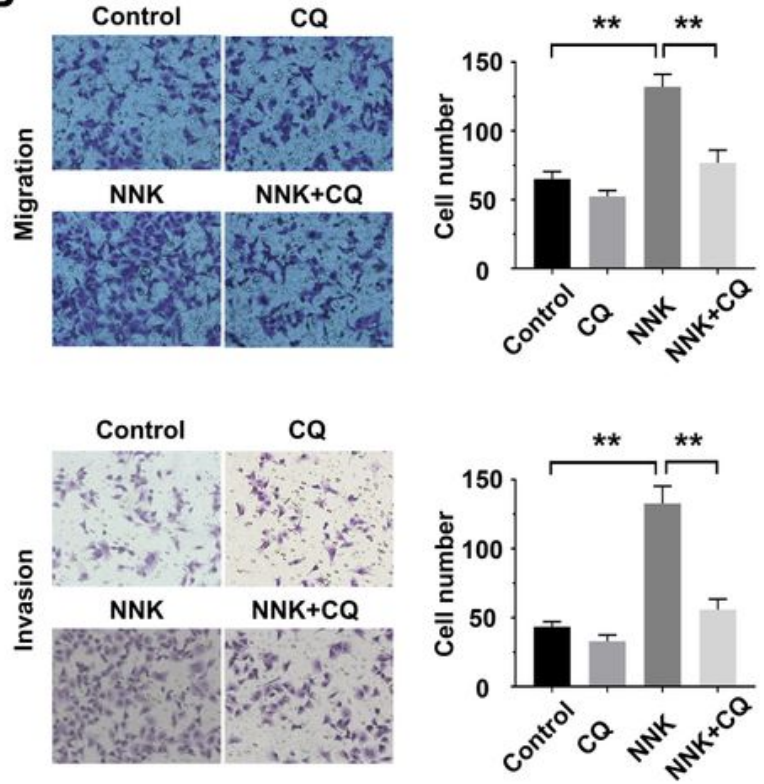

E

\# Gemcitabine

-F. Gemcitabine+CQ

-*. Gemcitabine+NNK

.... Gemcitabine+NNK+CQ
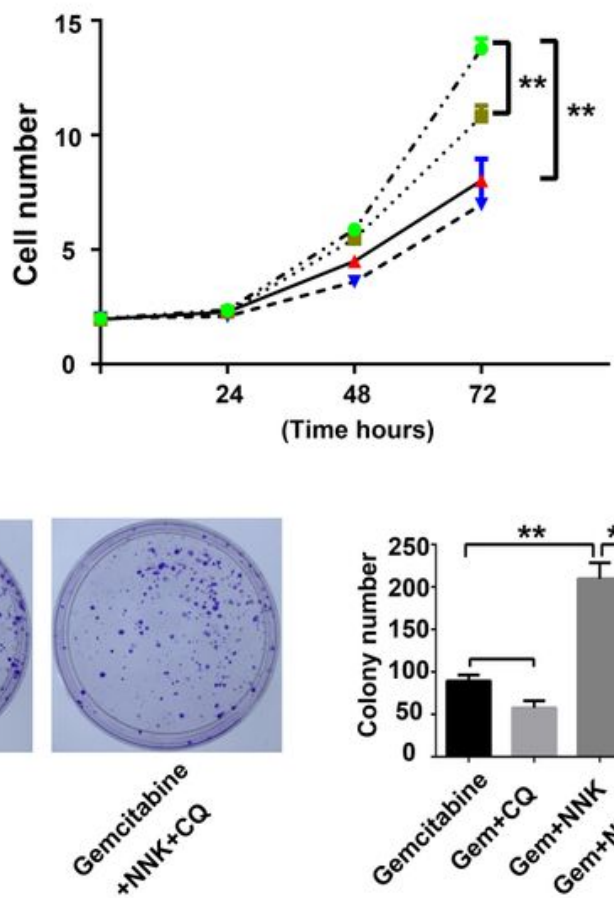


\section{Figure 3}

NNK enhances the stemness and chemoresistance of pancreatic cancer cells by activating autophagy. Colony formation assays (A), Transwell-based migration and invasion assays (B) and stem cell sphere formation assays (C) were performed in the control group, CQ group, NNK group and NNK plus CQ group of Panc-1 cells. (D) The mRNA levels of OCT-4, SOX-2 and Nanog were determined in the control group, CQ group, NNK group and NNK plus CQ group of Panc-1 cells. CCK-8 assays (E) and colony formation assays (F) were conducted in the gemcitabine group, gemcitabine plus CQ group, gemcitabine plus NNK group and gemcitabine plus CQ plus NNK group. $\left({ }^{*} p<0.05\right.$, $\left.{ }^{*} p<0.01\right)$.

A

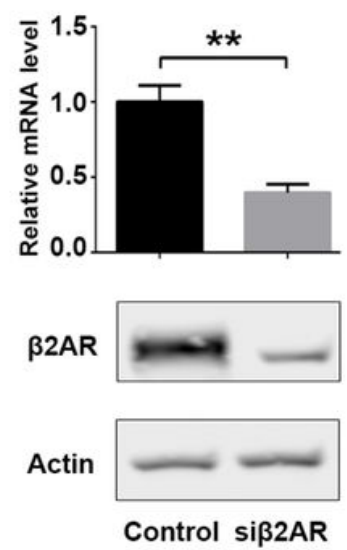

D

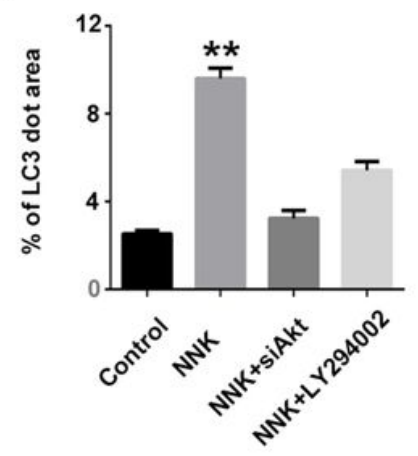

B

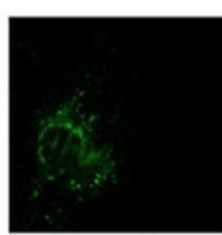

Control

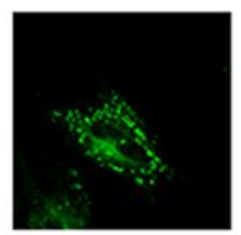

NNK

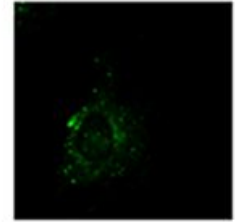

Siß2AR

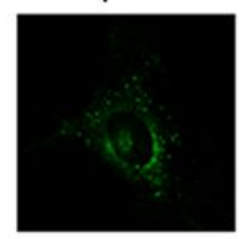

NNK+si $\beta 2 A R$

E

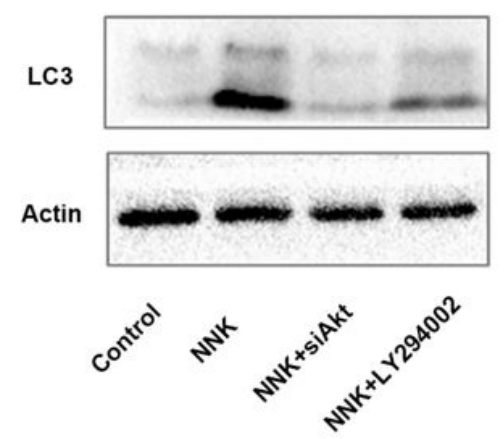

C
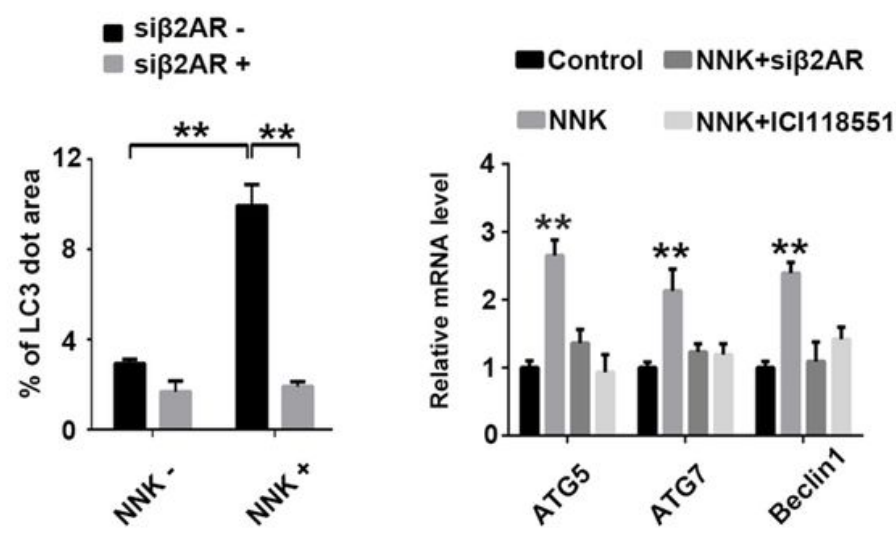

$\mathbf{F}$

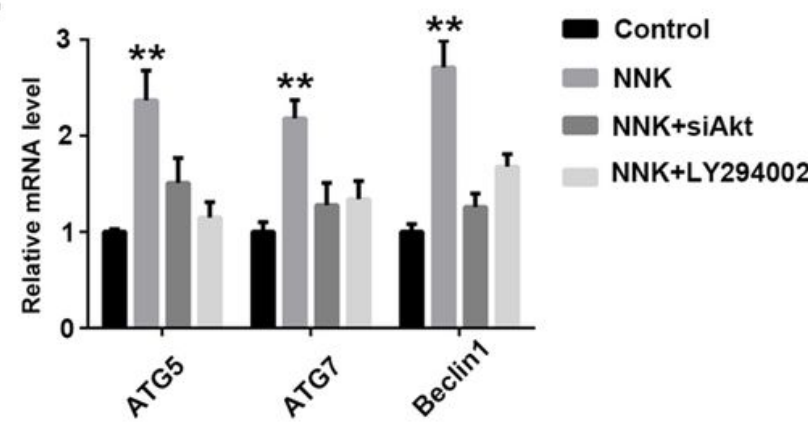

Figure 4

NNK promotes autophagy by modulating $\beta 2 A R$ and Akt. (A) The mRNA and protein levels of $\beta 2 A R$ were decreased by siRNA. (B) Immunofluorescence staining shows LC3 autophagy spots in the control group, siß2AR group, NNK group and siß2AR plus NNK group of Panc-1 cells, and a statistical graph is shown. (C) The mRNA levels of ATG5, ATG7 and Beclin1 were determined in the control group, NNK group, NNK group, siß2AR plus NNK group and ICl118551 plus NNK group. (D) LC3 autophagy spots were observed by immunofluorescence microscopy, and the results were graphed for the control group, NNK group, siAkt plus NNK group and LY 294002 plus NNK group of Panc-1 cells. (E)The protein level of LC3 in the control group, NNK group, siAkt plus NNK group and LY 294002 plus NNK group of Panc-1 cells was determined. 
(F) The mRNA levels of ATG5, ATG7 and Beclin1 in the control group, NNK group, siAkt plus NNK group and LY 294002 plus NNK group of Panc-1 cells were analysed.

A
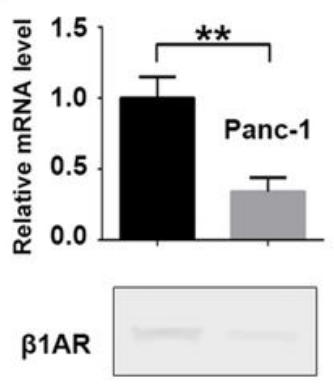

Actin

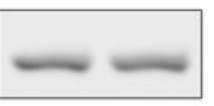

Control si $\beta 1 A R$

D
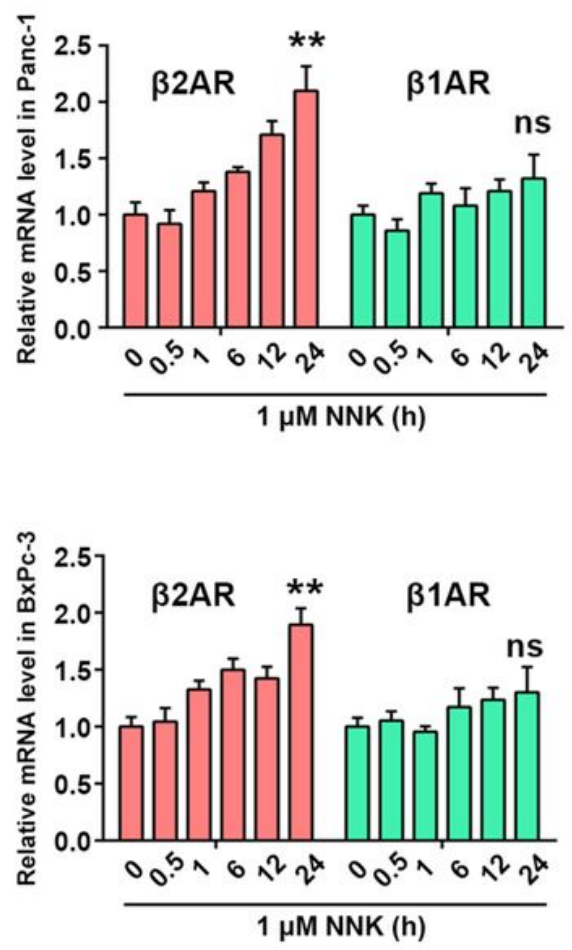

B

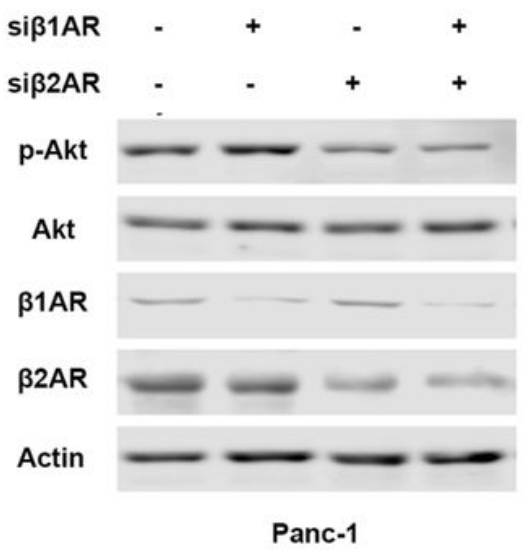

C

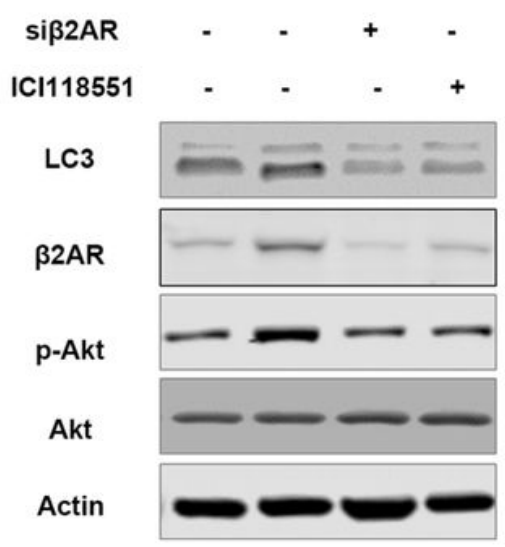

Panc-1

E

$\mathbf{F}$

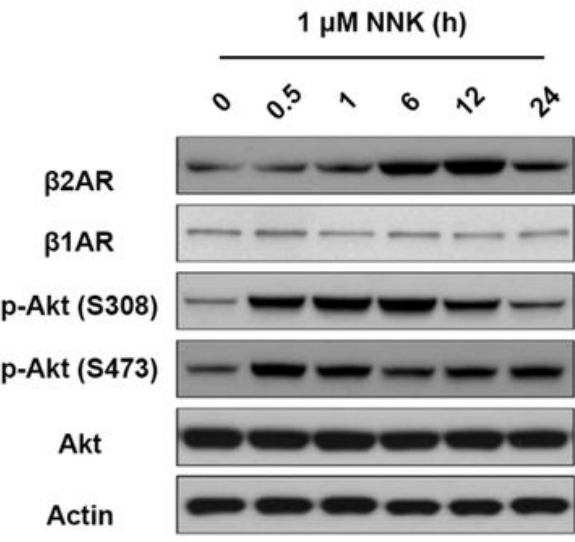

Panc-1
$1 \mu \mathrm{M}$ NNK (h)

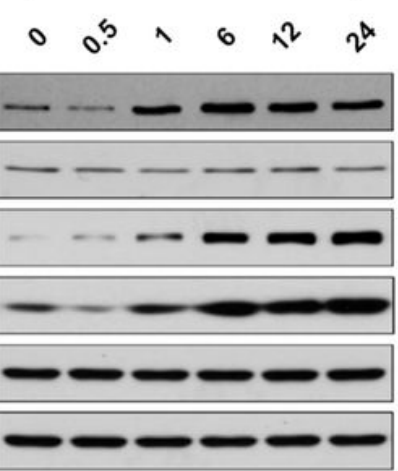

BxPc-3

\section{Figure 5}

NNK forms a potential $\beta 2 A R-A k t$ feedback loop in pancreatic cancer cells autophagy. (A) The mRNA and protein levels of $\beta 1 A R$ were decreased by siRNA. (B) The protein levels of $p$-Akt and Akt were assessed in the control group, siß1AR group, siß2AR group and siß1AR plus siß2AR group. (C) The protein levels of LC3, p-Akt and Akt were analysed in the control group, NNK group, NNK plus siß2AR group and NNK plus 
ICl118551 group. The mRNA and protein levels of $\beta 2 A R$, p-Akt and Akt were determined in Panc-1/BxPC-3 cells treated with NNK at different time periods. $\left({ }^{*} p<0.05,{ }^{* *} p<0.01\right)$. 